\title{
Epilepsy and inborn errors of metabolism in children
}

\author{
N. I. Wolf • A. García-Cazorla • G. F. Hoffmann
}

Received: 12 February 2009 /Submitted in revised form: 18 May 2009 / Accepted: 19 May 2009/Published online: 27 July 2009

(C) SSIEM and Springer 2009

\begin{abstract}
Summary Epilepsy is a frequent symptom in inborn errors of metabolism, with virtually no specific seizure types or EEG signatures. It is most important to look quickly for those few inborn errors of metabolism in which specific therapies such as supplementation of cofactors or diets can make all the difference. If these investigations remain negative, epilepsy has to be treated with conventional antiepileptic drugs. Still, epilepsy is a potentially treatable symptom of many inborn errors of metabolism, and optimal treatment is of great importance for patients and their families.
\end{abstract}

\section{Abbreviations \\ alpha-AASA alpha-aminoadipic semialdehyde CDG congenital disorders of glycosylation \\ GAMT guanidinoacetate methyltransferase}

Communicating editor: Jean-Marie Saudubray

Competing interests: None declared

In memory of Professor Robert Surtees our friend and outstanding colleague in the field of Paediatric Neurology and Neurometabolic Diseases.

N. I. Wolf $(\bowtie)$

Department of Child Neurology, VU University Medical Center (VUMC),

Postbus 7057, 1007 MB Amsterdam, The Netherlands

e-mail: n.wolf@vumc.nl

\section{A. García-Cazorla}

Department of Neurology, Hospital Sant Joan de Déu,

Barcelona, and Centre for Biomedical Research on Rare

Diseases (CIBER-ER), Instituto de Salud Carlos III,

Barcelona, Spain

G. F. Hoffmann

University Children's Hospital Heidelberg,

Heidelberg, Germany

\begin{tabular}{|c|c|}
\hline GCS & glycine cleavage system \\
\hline GLUT1 & glucose transporter type 1 \\
\hline EME & early myoclonic encephalopathy \\
\hline IEM & inborn error of metabolism \\
\hline MERRF & $\begin{array}{l}\text { myoclonic epilepsy with ragged red } \\
\text { fibres }\end{array}$ \\
\hline NCL & neuronal ceroid lipofuscinosis \\
\hline POLG1 & polymerase $\gamma 1$ \\
\hline RHADS & $\begin{array}{l}\text { rhythmic high-amplitude delta with } \\
\text { superimposed (poly)spikes }\end{array}$ \\
\hline
\end{tabular}

\section{Introduction}

Metabolic disorders are not a frequent cause of epilepsy. In many metabolic disorders though, epilepsy is a frequent symptom, e.g. untreated phenylketonuria, storage disorders, most organicacidopathies and urea cycle disorders. Sometimes epilepsy even dominates the clinical picture, especially in newborns and infants. Epilepsy may be so severe and deleterious for cognitive development that the term 'epileptic encephalopathy' can be used. In a few metabolic diseases, epilepsy responds to specific treatments based on diet or supplementation of cofactors (vitamin-responsive epilepsies), but for most of them specific treatment is unfortunately not available, and conventional antiepileptic drugs must be used, often with no satisfactory success. The type of epilepsy depends more on the age at presentation and the cortical areas involved than on the specific inborn error, although there are exceptions (Table 1). Isolated epilepsy without developmental delay, mental retardation, or other neurological symptoms or well defined epileptic syndromes do not suggest a metabolic disorder and 


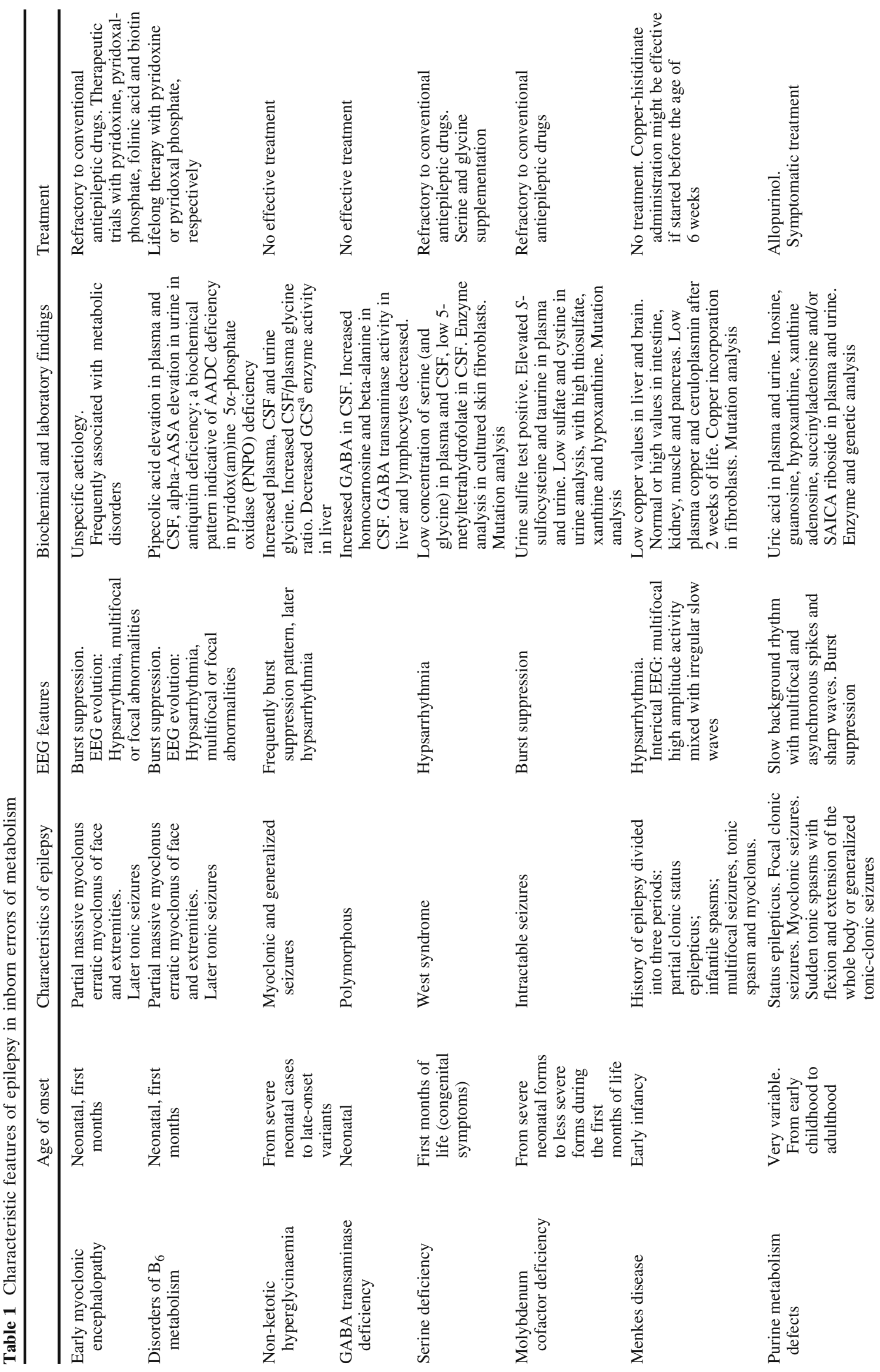



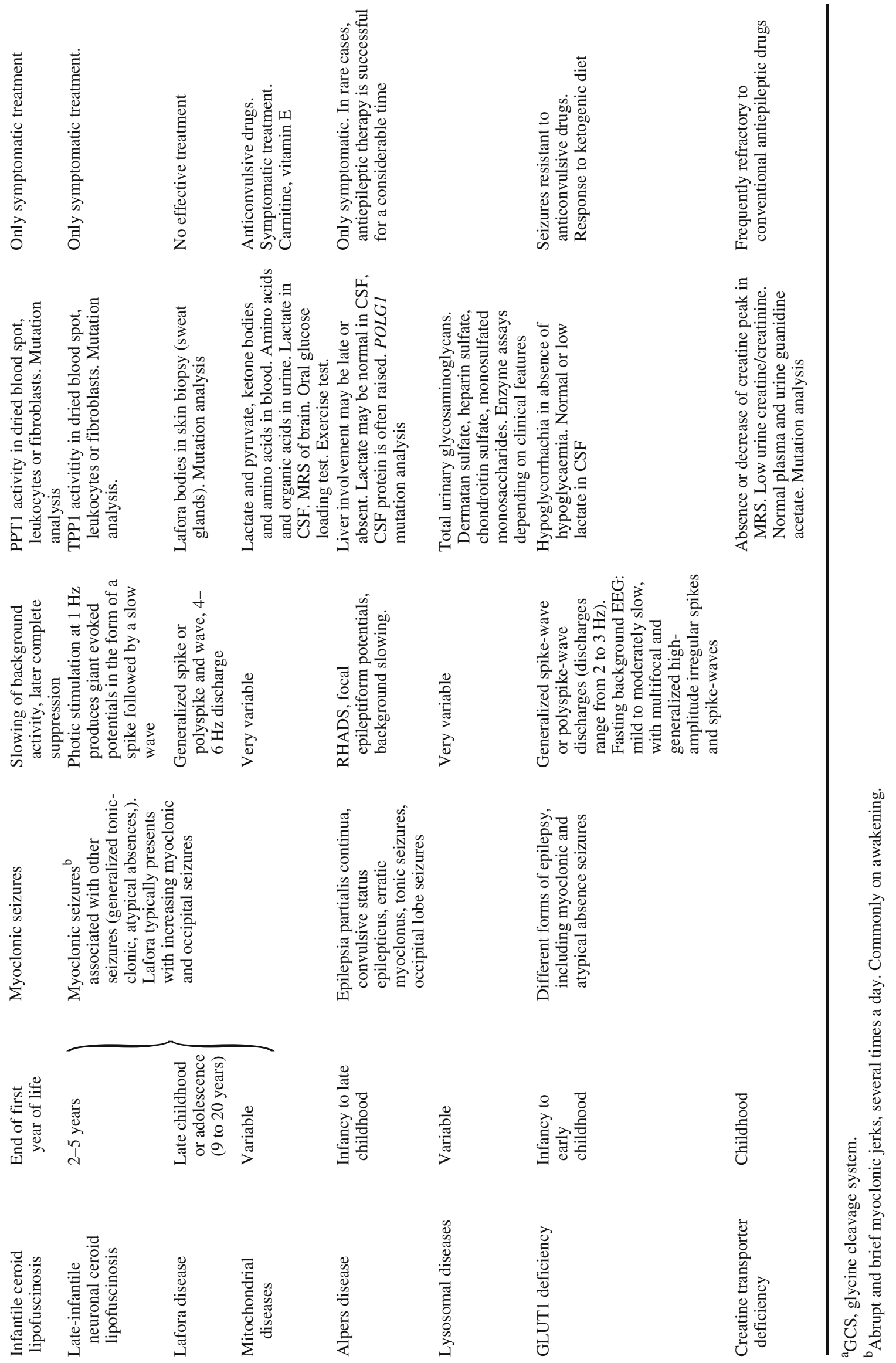
do not warrant metabolic investigations (Sedel et al 2007; Wolf et al 2005).

In most cases, epilepsy secondary to an IEM presents with polymorphic clinical and electroencephalographic features, making the classification as an epileptic syndrome difficult. Seizures are more frequently focal than generalized. Nevertheless, some types of seizures, such as myoclonic seizures, or certain electroencephalographic patterns, such as a burst-suppression pattern, suggest that metabolic disorders should be included early in the differential diagnosis. In the following we will concentrate on diseases and conditions where epilepsy is the predominant clinical manifestation and especially where the disease course can be positively influenced by specific metabolic therapies.

\section{Neonatal-onset epilepsy}

Always consider the possibility of an IEM in a neonate with unexplained and refractory epilepsy (BahiBuisson et al 2006b; Surtees and Wolf 2007). The great majority of vitamin-responsive epilepsies start in the neonatal period and early infancy and, although rare, are treatable disorders. A specific marker for pyridoxine-dependent epilepsy secondary to mutations in ALDH7A1 (Antiquitin) is alpha-aminoadipic semialdehyde (alpha-AASA) elevated in urine (Mills et al 2006); pipecolic acid is elevated in plasma and CSF (Plecko et al 2000). In pyridox(am)ine phosphate oxidase (PNPO) deficiency, affected neonates do not respond to treatment with pyridoxine but need pyridoxal phosphate for control of seizures. Biochemical abnormalities are secondary to impaired activities of the many enzymes needing pyridoxal phosphate as a cofactor and include slight elevations of glycine and threonine in CSF and plasma, an increase in the CSF concentration of 3-methoxytyrosine and a decrease in the CSF concentrations of 5-hydroxyindolacetic acid and homovanillic acid, the latter findings reflecting impaired activity of the aromatic amino acid decarboxylase (Mills et al 2005). It must be emphasized, however, that these biochemical abnormalities are not obligatory in PNPO deficiency (Hoffmann et al 2007). Treatment with pyridoxine and pyridoxal phosphate should be initiated as quickly as possible, without awaiting results of biochemical or genetic investigations as prognosis is better the earlier treatment is initiated. Infantile hypophosphatasia can also lead to pyridoxine-dependent seizures in neonates as pyridoxal phosphate is not dephosphorylated and therefore cannot cross membranes. Folinic acid-responsive seizures characterized by cessation of seizures after administration of folinic acid and unknown characteristic peaks in CSF monoamine analysis were long considered to be a separate entity. Only a few affected infants were published, and their pathogenesis could not be elucidated. Recently, however, it became clear that folinic acidresponsive seizures are also caused by mutations in $A L D H 7 A 1$ and therefore should be treated with adequate doses of pyridoxine. Whether additional treatment of these children with folinic acid is of added benefit, remains to be shown (Gallagher et al 2009).

In many cases of epilepsy with neonatal onset, the epilepsy syndrome can be defined as 'early myoclonic encephalopathy (EME)'. EME is characterized by partial, massive myoclonus, erratic myoclonus of face and extremities, tonic seizures refractory to conventional antiepileptic drugs, and burst-suppression or discontinuous pattern in the EEG (Fig. 1). Myoclonic jerks are often without EEG equivalent. EME can be due to vitamin-dependent diseases such as pyridoxineor pyridoxal phosphate-dependent seizures. Some disorders of amino acid metabolism such as nonketotic hyperglycinaemia, methylene tetrahydrofolate reductase (MTHFR) deficiency, GABA transaminase deficiency, serine deficiency, and congenital glutamine deficiency, can also give rise to this epileptic syndrome, each with its specific biochemical traits. In addition, defects of purine metabolism, sulfite oxidase deficiency, peroxisomal disorders and CDG syndromes should also be considered. In many cases of EME, the aetiology remains unclear despite adequate investigations, and prognosis is poor. In peroxisomal disorders as Zellweger syndrome, the main seizure type is focal clonic, epileptiform potentials and seizure patterns in the EEG are mainly seen at central and centroparietal regions, reflecting the underlying cortical abnormalities. Neonates with urea cycle defects can also present with seizures or status epilepticus.

\section{Epilepsy with onset in infancy}

Important treatable conditions at this age where epilepsy is the leading symptom are late-onset pyridoxine-dependent seizures, GLUT1 deficiency, biotinidase deficiency, serine deficiency and the group of creatine deficiency syndromes.

Patients with serine deficiency due to 3-phosphoglycerate dehydrogenase deficiency have congenital microcephaly. They develop severe psychomotor retardation with spastic tetraparesis and severe microcephaly. Seizures usually start in infancy as West syndrome with hypsarrhythmia (de Koning and Klomp 2004). Serine deficiency is most reliably diagnosed in CSF with 


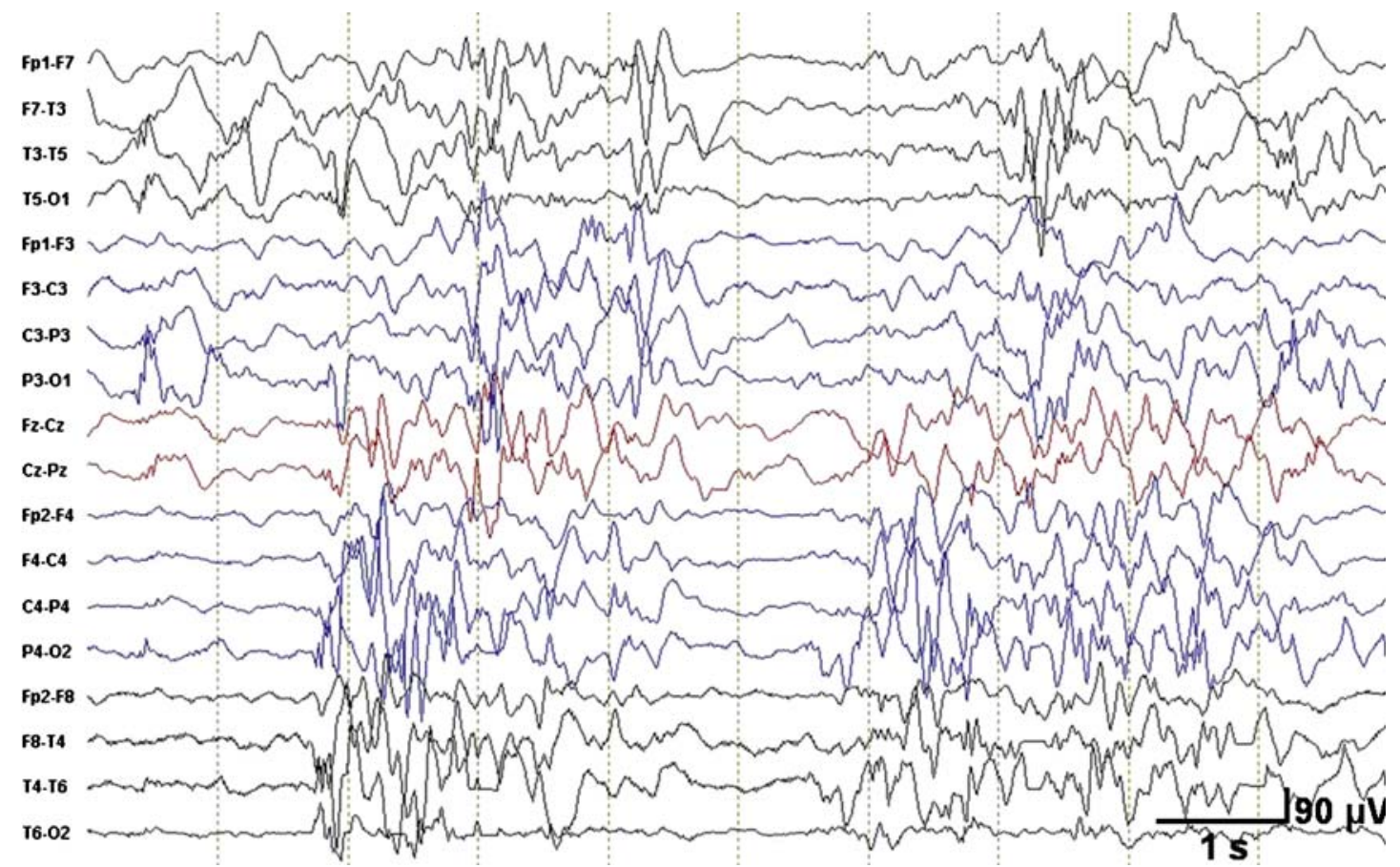

Fig. 1 Discontinuous activity and multifocal epileptiform potentials in a 6-month-old infant with pyridoxine-dependent epilepsy before starting pyridoxine

values $<14 \mu \mathrm{mol} / \mathrm{L}$ (normal CSF serine $42-86 \mu \mathrm{mol} / \mathrm{L}$ in infancy). Plasma levels can be normal. A very satisfactory outcome could be achieved by antenatal treatment in one patient (de Koning et al 2004).

GLUT1 deficiency is characterized by persistent hypoglycorrhachia in the absence of hypoglycaemia and with normal or low lactate in the CSF. Although the neurological phenotype may vary from case to case, epilepsy is the predominant feature in almost all patients (Seidner et al 1998). Clinical manifestations vary from mild to severe and include acquired microcephaly, developmental delay, pyramidal signs, a complex movement disorder with dystonia and ataxia, and different forms of seizures, including myoclonic seizures resistant to anticonvulsant drugs. GLUT1 deficiency can be effectively treated with the ketogenic diet. Generalized changes in the EEG can erroneously lead to the diagnosis of idiopathic generalized epilepsy (Fig. 2).

In biotinidase deficiency, epilepsy usually starts at 3 or 4 months of life. West syndrome is the most frequent epileptic type, and conventional antiepileptic drugs are ineffective. Alopecia and dermatitis can be useful clues, but are only present in less than $50 \%$ of patients. Low doses of biotin (5-10 mg/day) stop the seizures and prevent permanent sequels if started early enough (Salbert et al 1993).

Creatine is crucial for energy metabolism and is synthesized in a two-step process: the first is mediated by AGAT (arginine-glycine amidinotransferase), the second by GAMT (guanidinoacetate methyltransferase). Furthermore, a creatine transporter is required for creatine uptake into brain and muscle. Disorders of creatine synthesis or transport produce psychomotor delay and epilepsy that is frequently refractory to conventional antiepileptic drugs (Stockler et al 2007). Creatine transporter deficiency can give rise to generalized tonic-clonic as well as partial seizures. GAMT deficiency is associated with severe epilepsy including West syndrome and frequent episodes of nonconvulsive status epilepticus (Mercimek-Mahmutoglu et al 2006). Creatine supplementation, ornithine supplementation and an arginine-restricted diet are all important in controlling epilepsy in GAMT deficiency (Schulze et al 2001). An only partially treatable disorder is Menkes disease; epilepsy also starts in infancy, sometimes with prolonged clonic seizures (Bahi-Buisson et al 2006a).

\section{Epilepsy with onset in childhood and adolescence}

At this age, important disorders to consider are the neuronal ceroid lipofuscinoses (especially the lateinfantile, CLN2, and the juvenile, CLN3, forms) and mitochondrial disorders. Specific treatments are unfortunately not available, although conventional antiepileptic drug treatment can be used with some success and must be optimized. EEG is useful in lateinfantile NCL, since photic stimulation at $1 \mathrm{~Hz}$ pro- 


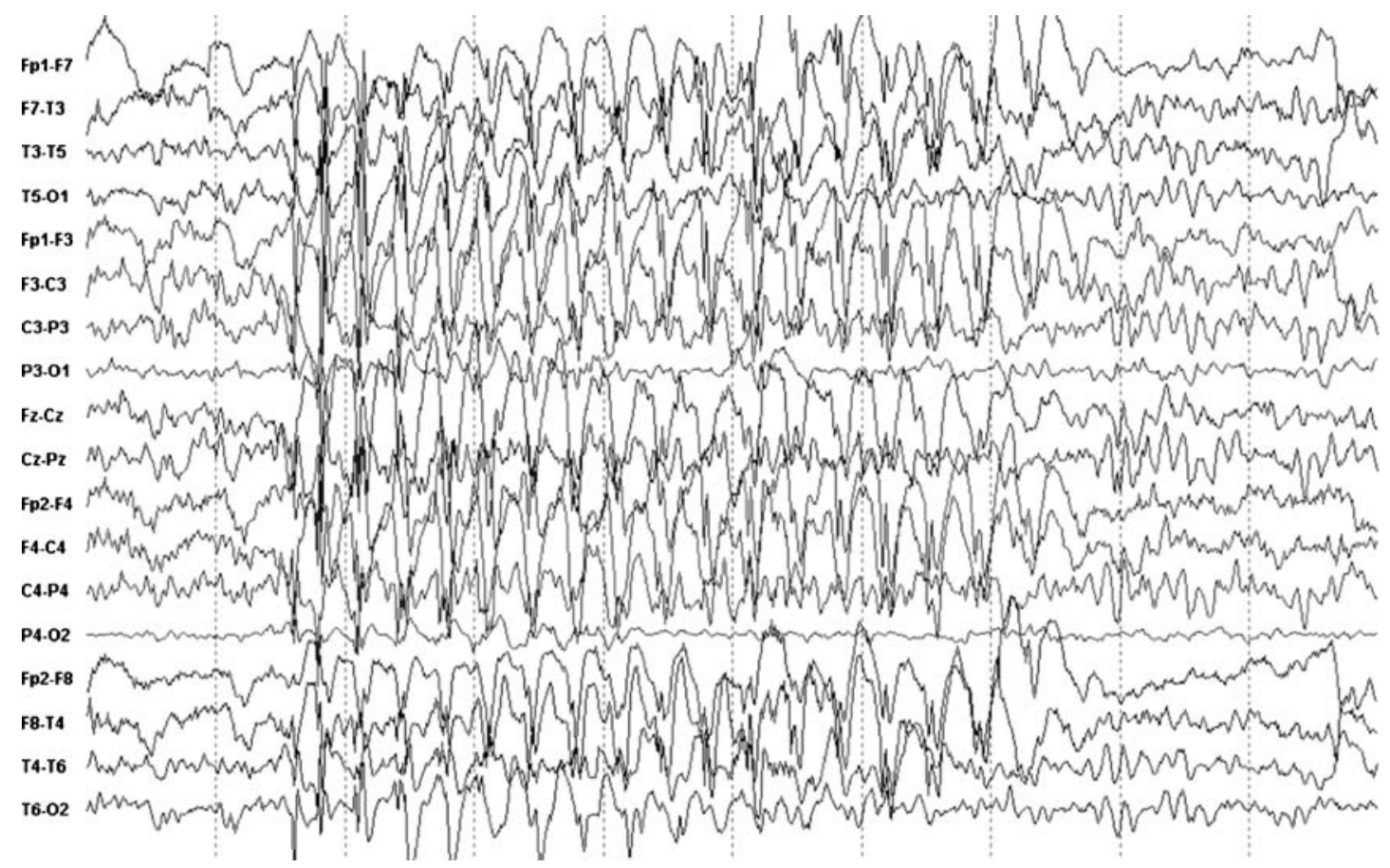

Fig. 2 Generalized 2.5/s spike wave paroxysm provoked by hyperventilation in a 16-year-old adolescent with GLUT1 deficiency

duces giant evoked potentials in the form of a spike followed by a slow wave (Fig. 3). Epilepsy with a variety of seizure types is also present in other storage disorders as Gaucher disease or Niemann-Pick type C disease. In the latter, attacks of gelastic cataplexy have to be differentiated from true epileptic seizures. The group of progressive myoclonic epilepsies usually starts in adolescence and comprises mitchochondrial disorders, especially MERRF, but also other disorders as Lafora body disease, CLN2, sialidosis type I and Unverricht-Lundborg disease. Appropriate genetic or histopathological testing is important if metabolic

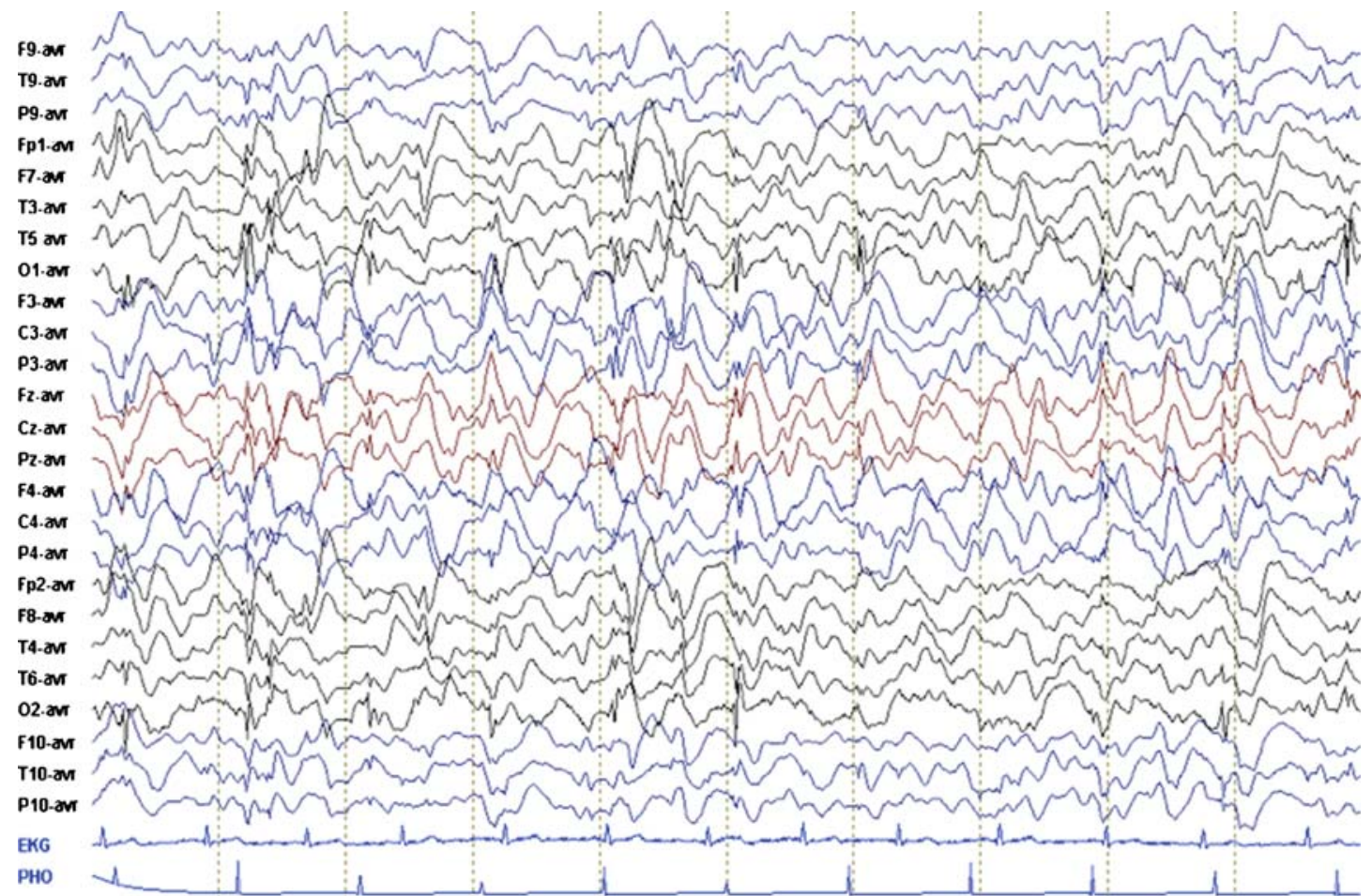

Fig. 3 EEG of a child with late-infantile neuronal ceroid lipofuscinosis. Photic stimulation at $1 \mathrm{~Hz}$ triggers posterior spike wave paroxysms 


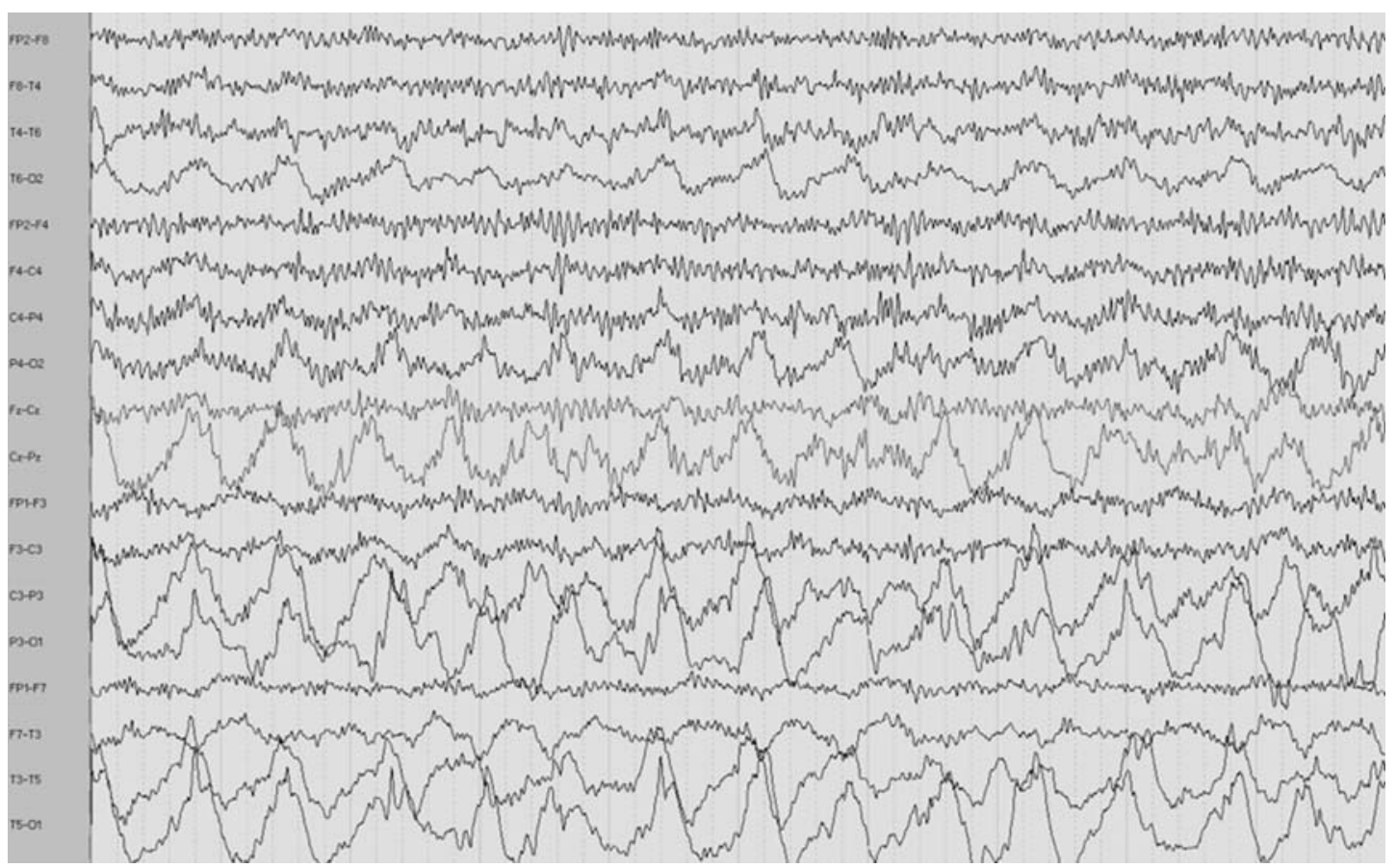

Fig. 4 Left posterior rhythmic high-amplitude delta with superimposed (poly)spikes (RHADS) in a child with status epilepticus and Alpers disease due to a homozygous mutation in POLG1

disorders have been ruled out. Succinic semialdehyde dehydrogenase (SSADH) deficiency leads in about half of the patients to epilepsy with tonic-clonic and absence-like seizures (Pearl et al 2003). Epilepsy is also part of the symptoms in hyperinsulinism/hyperammonaemia (HI/HA) syndrome and can present with myoclonic absences (Bahi-Buisson et al 2008).

Treatable disorders that may present in this age group with epilepsy are urea cycle disorders, especially in girls heterozygous for ornithine transcarbamylase deficiency, and defects of transsulfuration and remethylation, most importantly classical homocystinuria (Mudd et al 1985). Hyperprolinaemia type II leads to epilepsy in $>50 \%$ of patients by inactivation of pyridoxal phosphate due to pyrroline-5-carboxylate dehydrogenase deficiency, similarly as pyridoxine-dependent seizures due to Antiquitin deficiency. In a child with a seizure disorder, treatment with $5-30 \mathrm{mg} / \mathrm{kg}$ per day pyridoxine in a single dose should be started. The epilepsy mostly disappears in adulthood (Farrant et al 2001).

\section{Status epilepticus}

Convulsive status epilepticus is an emergency situation and should be treated according to local guidelines. It can occur in almost all inborn errors of metabolism where epilepsy is a feature. Usually this is not the first symptom of the disease. There is one exception: Alpers disease due to mutations in the gene for polymerase $\gamma 1$ (POLG1) can start with convulsive status epilepticus in a previously healthy child. As valproic acid can trigger fatal liver failure, this drug must not be used, although it is part of many treatment protocols for status epilepticus. Early suspicion and diagnosis are therefore important in this clinical setting; the EEG feature of posterior rhythmic highamplitude delta with superimposed (poly)spikes (RHADS, Fig. 4) is here very helpful although not mandatory in all cases (Wolf et al 2009).

\section{Principles of specific metabolic therapies}

High-dose substitution in vitamin-responsive epilepsies

- Pyridoxal phosphate: $30-40 \mathrm{mg} / \mathrm{kg}$ per day in at least three doses. To evaluate efficacy, therapy should be maintained for 5 days. Pyridoxal phosphate should also be effective in controlling pyridoxine-dependent seizures. It is advisable to obtain samples for appropriate biochemical tests before therapy, but treatment should not be delayed, nor should results be waited for. Pyridoxine- or pyridoxal phosphate-dependent seizures can be ascertained by molecular studies (Mills et al 2005, 2006).

- If pyridoxal phosphate is not available, pyridoxine should be used as first-line therapy: $100 \mathrm{mg}$ in a neonate (or $30 \mathrm{mg} / \mathrm{kg}$ ) either intravenously or 
orally in a single dose, preferably with EEG monitoring. There is no universal protocol for a pyridoxine trial. The dose of pyridoxine varies according to the physician's approach, but high doses may be necessary to control seizures, at least initially. In classical cases, we suggest a starting dose of $100 \mathrm{mg}$ intravenously. If there is no response within $24 \mathrm{~h}$, the same dose should be repeated (and possibly increased up to $500 \mathrm{mg}$ total) before excluding pyridoxine responsiveness. If there is uncertainty about a partial response, pyridoxine should be continued at $30 \mathrm{mg} / \mathrm{kg}$ per day for seven days before final conclusions are drawn.

The evaluation of pyridoxine- or pyridoxal phosphate-responsiveness of course does not preclude the introduction of other vitamins/drugs during this period of time if seizures do not stop. The possibility of lateonset pyridoxine-dependent seizures in children up to 3-4 years of age should be kept in mind and pyridoxal phosphate or pyridoxine may be tried. If one cares for a child whose epilepsy started in the neonatal period and has never been properly controlled and where it is not clear whether pyridoxine or pyridoxal phosphate have been given at all or in appropriate doses, this must be repeated.

- Biotin: 10-100 mg/day. Urinary organic acids and plasma biotinidase activity should be sampled before starting treatment

- Folinic acid: $2-3 \mathrm{mg} / \mathrm{kg}$ per day. It is recommended to maintain the treatment for one month to test efficacy. There is now evidence that at least some patients with folinic acid-responsive seizures do indeed have pyridoxine-responsive seizures due to mutations in ALDH7A1 (Gallagher et al 2009). Whether there is a distinct entity of 'true' folinic acid-responsive seizures remains to be shown. For children with Antiquitin deficiency, treatment with folinic acid in addition to pyridoxine might be beneficial (Gallagher et al 2009).

- Creatine: $300-400 \mathrm{mg} / \mathrm{kg}$ per day together with adequate dietary supplementations in case of GAMT deficiency. There is currently no possibility of treatment of male patients with creatine transporter deficiency.

- Ketogenic diet in GLUT-1 deficiency.

- In serine deficiency, L-serine should be administered orally (300-500 mg/kg per day) until normalization of plasma levels. If seizures persist, glycine should be added with up to $300 \mathrm{mg} / \mathrm{kg}$ per day.

\section{References}

Bahi-Buisson N, Kaminska A, Nabbout R, et al (2006a) Epilepsy in Menkes disease: analysis of clinical stages. Epilepsia 47: 380-386. doi:10.1111/j.1528-1167.2006.00432.x

Bahi-Buisson N, Mention K, Leger PL, et al (2006b) Neonatal epilepsy and inborn errors of metabolism. Arch Pediatr 13: 284-292. doi:10.1016/j.arcped.2005.10.024

Bahi-Buisson N, El Sabbagh S, Soufflet C, et al (2008) Myoclonic absence epilepsy with photosensitivity and a gain of function mutation in glutamate dehydrogenase. Seizure 17: 658-664. doi:10.1016/j.seizure.2008.01.005

de Koning TJ, Klomp LW (2004) Serine-deficiency syndromes. Curr Opin Neurol 17: 197-204. doi:10.1097/00019052200404000-00019

de Koning TJ, Klomp LW, van Oppen AC, et al (2004) Prenatal and early postnatal treatment in 3-phosphoglycerate-dehydrogenase deficiency. Lancet 364: 2221-2222. doi:10.1016/ S0140-6736(04)17596-X

Farrant RD, Walker V, Mills GA, Mellor JM, Langley GJ (2001) Pyridoxal phosphate de-activation by pyrroline-5carboxylic acid. Increased risk of vitamin $\mathrm{B}_{6}$ deficiency and seizures in hyperprolinemia type II. J Biol Chem 276: 15107-15116. doi:10.1074/jbc.M010860200

Gallagher RC, Van Hove JL, Scharer G, et al (2009) Folinic acid-responsive seizures are identical to pyridoxine-dependent epilepsy. Ann Neurol 65: 5550-556.

Hoffmann GF, Schmitt B, Windfuhr M, et al (2007) Pyridoxal 5 -phosphate may be curative in early-onset epileptic encephalopathy. $J$ Inherit Metab Dis 30: 96-99. doi:10.1007/s10545-008-9974-1

Mercimek-Mahmutoglu S, Stoeckler-Ipsiroglu S, Adami A, et al (2006) GAMT deficiency: features, treatment, and outcome in an inborn error of creatine synthesis. Neurology 67: 480 484. doi:10.1212/01.wnl.0000234852.43688.bf

Mills PB, Surtees RA, Champion MP et al (2005) Neonatal epileptic encephalopathy caused by mutations in the PNPO gene encoding pyridox(am)ine $5^{\prime}$-phosphate oxidase. Hum Mol Genet 14: 1077-1086. doi:10.1093/hmg/ddi120

Mills PB, Struys E, Jakobs C, et al (2006) Mutations in antiquitin in individuals with pyridoxine-dependent seizures. Nat Med 12: 307-309. doi:10.1038/nm1366

Mudd SH, Skovby F, Levy HL, et al (1985) The natural history of homocystinuria due to cystathionine beta-synthase deficiency. Am J Hum Genet 37: 1-31.

Pearl PL, Gibson KM, Acosta MT, et al (2003) Clinical spectrum of succinic semialdehyde dehydrogenase deficiency. Neurology 60: $1413-1417$.

Plecko B, Stockler-Ipsiroglu S, Paschke E, Erwa W, Struys EA, Jakobs C (2000) Pipecolic acid elevation in plasma and cerebrospinal fluid of two patients with pyridoxine-dependent epilepsy. Ann Neurol 48: 121-125. doi:10.1002/15318249(200007)48:1<121::AID-ANA20>3.0.CO;2-V

Salbert BA, Pellock JM, Wolf B (1993) Characterization of seizures associated with biotinidase deficiency. Neurology 43: $1351-1355$.

Schulze A, Ebinger F, Rating D, Mayatepek E (2001) Improving treatment of guanidinoacetate methyltransferase deficiency: reduction of guanidinoacetic acid in body fluids by arginine restriction and ornithine supplementation. Mol Genet Metab 74: 413-419. doi:10.1006/ mgme.2001. 3257 
Sedel F, Gourfinkel-An I, Lyon-Caen O, Baulac M, Saudubray JM, Navarro V (2007) Epilepsy and inborn errors of metabolism in adults: a diagnostic approach. $J$ Inherit Metab Dis 30: 846-854. doi:10.1007/s10545-007-0723-7

Seidner G, Alvarez MG, Yeh JI, et al (1998) GLUT-1 deficiency syndrome caused by haploinsufficiency of the blood-brain barrier hexose carrier. Nat Genet 18: 188-191. doi:10.1038/ ng0298-188

Stockler S, Schutz PW, Salomons GS (2007) Cerebral creatine deficiency syndromes: clinical aspects, treatment and path- ophysiology. Subcell Biochem 46: 149-166. doi:10.1007/9781-4020-6486-9_8

Surtees R, Wolf N (2007) Treatable neonatal epilepsy. Arch Dis Child 92: 659-661. doi:10.1136/adc.2007.116913

Wolf NI, Bast T, Surtees R (2005) Epilepsy in inborn errors of metabolism. Epileptic Disord 7: 67-81.

Wolf NI, Rahman S, Schmitt B, et al (2009) Status epilepticus in children with Alpers' disease caused by POLG1 mutations: EEG and MRI features. Epilepsia 50: 1596-1607. doi:10.1111/j.1528-1167.2008.01877.x 